\title{
Laws and identities for some upper triangular matrices
}

\author{
T. Rashkova and A. Mihova
}




\title{
LAWS AND IDENTITIES FOR SOME UPPER TRIANGULAR MATRICES
}

\author{
T. RASHKOVA AND A. MIHOVA
}

Received 17 December, 2008

\begin{abstract}
J. C. Robson has investigated the ideal $I_{n}$ of all polynomials in the free associative algebra $R\langle x\rangle$ over a non-commutative ring $R$ generated by $x$ and the $n^{2}$ entries of an $n \times n$ matrix $\alpha=\left(a_{i j}\right)$, which are satisfied by $\alpha$. He found four cubics generating the ideal for $n=$ 2 and proved its finite generation for any $n$. Ts. Rashkova has considered the ideal $I_{2}$ for matrix algebras with involution over a noncommutative ring and over a field of characteristic zero. In the paper the ideal $I_{3}$ is described for some special upper triangular matrices over a field of characteristic 0 . The $T$-ideal $T\left(U_{2}(G)\right)$ is investigated as well for $G$ denoting the infinite dimensional Grassmann algebra.
\end{abstract}

2000 Mathematics Subject Classification: 16R50, 16R10

Keywords: laws, identities, Robson cubics

\section{INTRODUCTION}

The Cayley-Hamilton theorem and the corresponding trace identity play a fundamental role in proving classical results about the polynomial and trace identities of the $n \times n$ matrix algebra $M_{n}(K)$ over a field $K$ of characteristic zero.

The structure theory of semisimple rings and quantum matrices for example show the importance of matrices over non-commutative rings in the theory of PI-algebras and other branches of algebra as well.

J. C. Robson investigated in [12-14] the ideal $I_{n}$ of all polynomials (including nonmonics) in the free associative algebra $R\langle x\rangle$ over a non-commutative ring $R$ generated by $x$ and the $n^{2}$ entries of an $n \times n$ matrix $\alpha=\left(a_{i j}\right)$, which are satisfied by $\alpha$. Those polynomials we call the laws over $R$ of a non-commutative $n \times n$ matrix $\alpha$. These are not polynomial identities since the entries of $\alpha$ are allowed as coefficients in the laws and they vary with the choice of $\alpha$.

Robson showed that $I_{n}$ is an insertive ideal (meaning that its homogeneous elements are closed under inner multiplication by a constant $a_{i j}$ in some fixed position) and as such is finitely generated (see [13] and [12, Theorem 2.3]).

The research of the first author was supported in part by the Bulgarian Scientific Research Fund, Grant No. MM1503/2005. 
The minimal degree of polynomials in $I_{n}$ remains unknown. However, for the case $n=2$, Robson [12, Proposition 3.2] found four polynomials of degree 3 (least possible) in $I_{2}$ and Pearson showed in [10, Corollary] that these four Robson cubics do indeed generate $I_{2}$ as an insertive ideal.

\section{RESULtS}

The first study of the non-commutative case for $n=3$ was done in [9]. The results there provide further evidence of the tantalizing complexity of even these small matrices. Each of the four found laws of degree 7 has 1156 terms. Thus, special cases of $3 \times 3$ matrices even over a field are of interest.

In [11] we study the ideal $I_{3}$ for some special $3 \times 3$ upper triangular matrices considered in [7]. These algebras could be endowed with involution $*$ and their $*$ codimensions have important properties.

Here we give a complete answer for the existing laws in these algebras and investigate the $T$-ideal $T\left(U_{2}(G)\right)$ of the $2 \times 2$ upper triangular matrices over $G$, where $G$ stands for the infinite dimensional Grassmann algebra.

\subsection{Laws for upper triangular matrices over a field}

\subsubsection{Special upper triangular matrices}

In [7] D. La Mattina and P. Misso study some associative algebras with involution generating *-varieties of algebras with linear or linearly bounded sequences of $*$ codimensions. Questions concerning laws for them were discussed in [11]. Here we give the complete answers.

Let $K$ be a field of characteristic zero and

$$
M_{2}(K)=\left\{x=\left(\begin{array}{ccc}
a & b & c \\
0 & a & b \\
0 & 0 & a
\end{array}\right): a, b, c \in K\right\} .
$$

All coefficients in the polynomials below mean the corresponding scalar matrix, i. e., $a=a E$ for example.

Theorem 1. The only Robson cubic for a matrix from $M_{2}(K)$ in the general case is $r(x)=(x-a)^{3}$. For $b=0, c \neq 0$ a law of minimal degree is $(x-a)^{2}$ and for $b=c=0$ it is $x-a$.

Proof. For a matrix $x$ from the considered algebra we prove directly that $(x-$ $a)^{3}=0$. Due to $[8$, Lemma 2, p.432] considering the subring $T$ of the diagonal elements of a generic upper triangular matrix $A$ and $w(x) \in T\langle x\rangle$, then $w(A)=0$ iff $w(x) \in\left\langle x-a_{11}\right\rangle\left\langle x-a_{22}\right\rangle \cdots\left\langle x-a_{n n}\right\rangle$, where $\langle f\rangle$ is the ideal generated by $f$. In the cited Lemma $T$ is a subring of a non-commutative ring. In Theorem 1 the elements of the matrix are elements of a field and then $T\langle x\rangle=K\langle x\rangle$ and the possible 
laws of degree less or equal to 3 could be given with the linear combination

$$
A=\alpha_{1}+\alpha_{2} x+\alpha_{3}(x-a)+\alpha_{4} x^{2}+\alpha_{5}(x-a)^{2}+\alpha_{6} x(x-a) .
$$

Equating to zero the corresponding entries we get the system

$$
\begin{aligned}
\alpha_{1}+a \alpha_{2}+a^{2} \alpha_{4} & =0, \\
b \alpha_{2}+b \alpha_{3}+2 a b \alpha_{4}+a b \alpha_{6} & =0, \\
c \alpha_{2}+c \alpha_{3}+\left(2 a c+b^{2}\right) \alpha_{4}+b^{2} \alpha_{5}+\left(a c+b^{2}\right) \alpha_{6} & =0 .
\end{aligned}
$$

For $b \neq 0$ the system has a solution

$$
\begin{aligned}
& \alpha_{5}=-\alpha_{4}-\alpha_{6}, \\
& \alpha_{1}=a \alpha_{3}+a^{2} \alpha_{4}+a^{2} \alpha_{6}, \\
& \alpha_{2}=-\alpha_{3}-2 a \alpha_{4}-a \alpha_{6} .
\end{aligned}
$$

In this case $A$, is identically equal to zero. Considering $b=0$, we get the rest of the statement.

Let us put

$$
M_{3}(K)=\left\{x=\left(\begin{array}{ccc}
a & b & c \\
0 & 0 & d \\
0 & 0 & a
\end{array}\right): a, b, c, d \in K\right\} .
$$

Theorem 2. The only Robson cubic for a matrix from $M_{3}(K)$ in the general case is $r(x)=(x-a)^{2} x$. For the three cases $b \neq 0, c=d=0 ; d \neq 0, b=c=0$, and $b=c=d=0$ a law of minimal degree is $(x-a) x$.

Proof. Directly we calculate that for a matrix $x$ from the considered algebra we have $(x-a)^{2} x=0$. Then, due to Lemma 2 from [8, p. 432], considerations analogous to the proof of Theorem 1 lead us to the linear combination

$$
A=\alpha_{1}+\alpha_{2} x+\alpha_{3}(x-a)+\alpha_{4} x^{2}+\alpha_{5}(x-a)^{2}+\alpha_{6} x(x-a)
$$

of all possible laws of degree $\leq 3$. Equating to zero the corresponding entries, we obtain the system

$$
\begin{aligned}
\alpha_{1}+a \alpha_{2}+a^{2} \alpha_{4} & =0 \\
b \alpha_{2}+b \alpha_{3}+a b \alpha_{4}-a b \alpha_{5} & =0 \\
c \alpha_{2}+c \alpha_{3}+(2 a c+b d) \alpha_{4}+b d \alpha_{5}+(a c+b d) \alpha_{6} & =0 \\
\alpha_{1}-a \alpha_{3}+a^{2} \alpha_{5} & =0 \\
d \alpha_{2}+d \alpha_{3}+a d \alpha_{4}-a d \alpha_{5} & =0
\end{aligned}
$$


For $b \neq 0, d \neq 0$ the system has a solution

$$
\begin{aligned}
& \alpha_{6}=-\alpha_{4}-\alpha_{5}, \\
& \alpha_{1}=a \alpha_{3}-a^{2} \alpha_{5}, \\
& \alpha_{2}=-\alpha_{3}-a \alpha_{4}+a \alpha_{5} .
\end{aligned}
$$

In this case $A$ is identically equal to zero.

The three cases $b \neq 0, c=d=0 ; d \neq 0, b=c=0$, and $b=c=d=0$ give the law $(x-a) x$.

Let us put

$$
M_{4}(K)=\left\{x=\left(\begin{array}{ccc}
0 & b & c \\
0 & a & d \\
0 & 0 & 0
\end{array}\right): a, b, c, d \in K\right\} .
$$

Theorem 3. The only Robson cubic for a matrix from $M 4(K)$ in the general case is $r(x)=(x-a) x^{2}$. For $c=d=0$ or $b=c=0$ a law of minimal degree is $(x-a)$.

Proof. It follows the above pattern of proof. Directly we get $(x-a) x^{2}=0$. The corresponding system is

$$
\begin{aligned}
\alpha_{1}+a \alpha_{2}+a^{2} \alpha_{4} & =0, \\
b \alpha_{2}+b \alpha_{3}+a b \alpha_{4}-a b \alpha_{5} & =0, \\
c \alpha_{2}+c \alpha_{3}+b d \alpha_{4}+(b d-2 a c) \alpha_{5}+(b d-a c) \alpha_{6} & =0, \\
\alpha_{1}-a \alpha_{3}+a^{2} \alpha_{5} & =0, \\
d \alpha_{2}+d \alpha_{3}+a d \alpha_{4}-a d \alpha_{5} & =0 .
\end{aligned}
$$

For $b \neq 0, d \neq 0$ the system has the same solution as in the previous theorem and $A$ is identically equal to zero.

The two cases $c=d=0$ and $b=c=0$ give the law $(x-a) x$.

\subsubsection{The general upper triangular case}

Now we consider the general case, namely, the algebra $U_{3}(K, *)$ of the upper triangular matrices of order 3 with the involution $*$ reflection along the second diagonal. We could find the analogues of the Robson cubics for the $*$-symmetric matrices and for the $*$-skew-symmetric matrices as well.

Theorem 4. The only law of minimal degree for a symmetric matrix $x \in U_{3}^{+}(K, *)$, where

$$
U_{3}^{+}(K, *)=\left\{x=\left(\begin{array}{ccc}
a & b & d \\
0 & c & b \\
0 & 0 & a
\end{array}\right): a, b, c \in K\right\},
$$

is $(x-a)^{2}(x-c)$. For $b=d=0$ it is $(x-a)(x-c)$. 
Proof. Directly we calculate that $(x-a)^{2}(x-c)=0$. Then as explained in the above proofs we have to form the linear combination

$$
A=\alpha_{1}+\alpha_{2}(x-a)+\alpha_{3}(x-a)^{2}+\alpha_{4}(x-c)+\alpha_{5}(x-c)^{2}+\alpha_{6}(x-a)(x-c)
$$

of all possible laws of degree $\leq 3$. Equating to zero the corresponding entries we get the system

$$
\begin{aligned}
\alpha_{1}+(a-c) \alpha_{4}+(a-c)^{2} \alpha_{5} & =0, \\
b \alpha_{2}+b(c-a) \alpha_{3}+b \alpha_{4}+b(a-c) \alpha_{5} & =0, \\
d \alpha_{2}+b^{2} \alpha_{3}+d \alpha_{4}+\left(2 d(a-c)+b^{2}\right) \alpha_{5}+\left(d(a-c)+b^{2}\right) \alpha_{6} & =0, \\
\alpha_{1}+(c-a) \alpha_{2}+(c-a)^{2} \alpha_{3} & =0, \\
b \alpha_{2}+b(c-a) \alpha_{3}+b \alpha_{4}+b(a-c) \alpha_{5} & =0, \\
\alpha_{1}+(a-c) \alpha_{4}+(a-c)^{2} \alpha_{5} & =0 .
\end{aligned}
$$

For $b \neq 0, d \neq 0$ the system has a solution

$$
\begin{aligned}
& \alpha_{6}=-\alpha_{3}-\alpha_{5}, \\
& \alpha_{1}=-(a-c) \alpha_{4}-(a-c)^{2} \alpha_{5}, \\
& \alpha_{2}=-(-a+c) \alpha_{3}-\alpha_{4}-(a-c) \alpha_{5} .
\end{aligned}
$$

In this case $A$, is identically equal to zero. For $b=d=0$, we obtain the quadratic law $(x-a)(x-c)$.

We point that in the general case $(x-a)^{2}(x-c)=0$ is in fact the CayleyHamilton theorem in a factor form, i. e., $x^{3}-(2 a+c) x^{3}+a(a+2 c) x-a^{2} c=0$.

Theorem 5. The only law of minimal degree for a skew-symmetric matrix $x \in$ $U_{3}^{-}(K, *)$, where

$$
U_{3}^{-}(K, *)=\left\{x=\left(\begin{array}{ccc}
a & b & 0 \\
0 & c & -b \\
0 & 0 & -a
\end{array}\right): a, b, c \in K\right\},
$$

is $(x-a)(x-c)(x+a)$. If $a=b=0$, then a law is $x(x-c)$.

Proof. The law $(x-a)(x-c)(x+a)=0$ is checked directly. Then we form the linear combination

$$
\begin{aligned}
A= & \alpha_{1}+\alpha_{2}(x-a)+\alpha_{3}(x-a)^{2}+\alpha_{4}(x-c) \\
& +\alpha_{5}(x-c)^{2}+\alpha_{6}(x+a)+\alpha_{7}(x+a)^{2} \\
& +\alpha_{8}(x-a)(x-c)+\alpha_{9}(x-a)(x+a)+\alpha_{10}(x-c)(x+a)
\end{aligned}
$$


of all possible laws of degree $\leq 3$. Equating to zero the corresponding entries, we obtain the system

$$
\begin{aligned}
\alpha_{1}+(a-c) \alpha_{4}+(a-c)^{2} \alpha_{5}+2 a \alpha_{6}+4 a^{2} \alpha_{7}+2 a(a-c) \alpha_{10} & =0, \\
b \alpha_{2}+b(c-a) \alpha_{3}+b \alpha_{4}+b(a-c) \alpha_{5}+b \alpha_{6} & \\
+b(c+3 a) \alpha_{7}+b(c+a) \alpha_{9}+2 a b \alpha_{10} & =0, \\
-b^{2} \alpha_{3}-b^{2} \alpha_{5}-b^{2} \alpha_{7}-b^{2} \alpha_{8}-b^{2} \alpha_{9}-b^{2} \alpha_{10} & =0, \\
\alpha_{1}+(c-a) \alpha_{2}+(c-a)^{2} \alpha_{3}+(c+a) \alpha_{6}+(c+a)^{2} \alpha_{7}+(c-a)(c+a) \alpha_{9} & =0, \\
-b \alpha_{2}-b(c-3 a) \alpha_{3}-b \alpha_{4}+b(a+c) \alpha_{5}-b \alpha_{6} & \\
-b(c+a) \alpha_{7}+2 a b \alpha_{8}-b(c-a) \alpha_{9} & =0, \\
\alpha_{1}-2 a \alpha_{2}+4 a^{2} \alpha_{3}-(a+c) \alpha_{4}+(a+c)^{2} \alpha_{5}+2 a(a+c) \alpha_{8} & =0 .
\end{aligned}
$$

Its solution is

$$
\begin{aligned}
& \alpha_{1}=-2 a(a-c) \alpha_{10}-(a-c) \alpha_{4}-(a-c)^{2} \alpha_{5}-2 a \alpha_{6}-4 a^{2} \alpha_{7}, \\
& \alpha_{8}=-\alpha_{10}-\alpha_{3}-\alpha_{5}-\alpha_{7}-\alpha_{9}, \\
& \alpha_{2}=-2 a \alpha_{10}-(-a+c) \alpha_{3}-\alpha_{4}-(a-c) \alpha_{5}-\alpha_{6}-(3 a+c) \alpha_{7}-(a+c) \alpha_{9} .
\end{aligned}
$$

In this case $A$, is identically equal to zero. The case $a=b=0$ leads one directly to the validity of the law $x(x-c)$.

The law in the general case illustrates the Cayley-Hamilton theorem in a factor form, namely $x^{3}-c x^{2}-a^{2} x+a^{2} b=0$. All the computations are made using the computer algebra system Mathematica.

\subsection{Laws and identities for upper triangular matrices over the Grassmann alge-} bra

\subsubsection{Preliminaries}

We consider the matrix algebra of the $2 \times 2$ upper triangular matrices $U_{2}(G)$ over the Grassmann algebra $G$.

We recall the definition of the infinite dimensional Grassmann algebra $G$, namely,

$$
G=G(V)=K\left\langle v_{1}, v_{2}, \ldots \mid v_{i} v_{j}+v_{j} v_{i}=0, i, j=1,2, \ldots\right\rangle .
$$

The algebra $G^{\prime}$ (without 1 ) has a basis $v_{i_{1}} v_{i_{2}} \ldots v_{i_{k}}$, where $1 \leq i_{1}<i_{2}<\ldots<i_{k}$. The elements $v_{i}$ are called generators of $G^{\prime}$ while the elements $v_{i_{1}} v_{i_{2}} \ldots v_{i_{k}}$ for $1 \leq$ $i_{1}<i_{2}<\ldots<i_{k}$ are called basic monomials of $G^{\prime}$. For $G=G^{\prime} \cup 1$, a generator is 1 as well. The algebras $G$ and $G^{\prime}$ are PI-equivalent (they satisfy one and the same identities). It is easy to be seen that $G^{\prime}=J(G)$ for $J(G)$ being the Jacobson radical of the algebra. 
The algebra $G$ is in the mainstream of recent research in PI theory. Its importance is connected mainly with the structure theory for the $T$-ideals of identities of associative algebras developed by Kemer in [5]. Kemer proved [5, Theorem 1.2] that any $T$-prime $T$-ideal can be obtained as the $T$-ideal of identities of one of the following algebras: $M_{n}(K), M_{n}(G)$ and $M_{n, u}(G)$, the latter being the algebra of $n \times n$ supermatrices over $G=G_{0} \oplus G_{1}$ with $G_{0}$ blocks (with entries of even degree) of sizes $u \times u$ and $(n-u) \times(n-u)$ and with $G_{1}$ blocks (with entries of odd degree) of sizes $u \times(n-u)$ and $(n-u) \times u$.

Well known facts concerning the algebra $G$ are the following:

Proposition 1 ([6, Corollary, p. 437]). The T-ideal $T(G)$ is generated by the identity $\left[x_{1}, x_{2}, x_{3}\right]=0$.

Proposition 2 ([4, Exercise 5.3]). For $G_{k}=G\left(V_{k}\right)$ over $k$-dimensional vector space $V_{k}$ all identities follow from the identity $\left[x_{1}, x_{2}, x_{3}\right]=0$ and the standard identity $S_{2 p}\left(x_{1}, \ldots, x_{2 p}\right)=\sum_{\sigma \in \operatorname{Sym}(2 p)}(-1)^{\sigma} x_{\sigma(1)} \ldots x_{\sigma(2 p)}=0$, where $p$ is the minimal integer with $2 p>k$.

Remark 1. In the monograph [4, Exercise 5.3] one could see that the identity $\left[x_{1}, x_{2}\right] \ldots\left[x_{2 p+1}, x_{2 p+2}\right]=0$ on $G_{2 p+1}$ is equivalent to the standard identity of degree $2 p+2$.

Remark 2. It could be seen [4, Exercise 5.8] that the $T$-ideal of the algebra $M_{2}(K)$ from Theorem 1 is generated by the identities $\left[x_{1}, x_{2}, x_{3}\right]=0$ and $S_{4}\left(x_{1}, \ldots, x_{4}\right)=0$. Nevertheless, the algebra $M_{2}(K)$ is not isomorphic to the Grassmann algebra $G_{2}$ of the two-dimensional vector space.

For the rest of the paper we will use capital letters for the matrices with entries from the Grassmann algebra.

\subsubsection{The $T$-ideal $T\left(U_{2}(G)\right)$}

Theorem 6. The identity $\left[X_{1}, X_{2}, X_{3}\right]\left[X_{4}, X_{5}, X_{6}\right]=0$ holds on the algebra $U_{2}(G)$.

Proof. As the considered polynomial is multilinear we could rely on [16, Remark 3.1] stating that it is an identity on $M_{2}(G)$ if and only if for every choice of the matrix units $e_{a_{i}, b_{i}}$ and either $v_{i}^{*}=v_{i}$ or $v_{i}^{*}=1$, the substitution $x_{i} \rightarrow e_{a_{i}, b_{i}} v_{i}^{*}$ in the polynomial gives zero.

We take the matrices $X_{i}=\left(\begin{array}{cc}a_{1 i} & b_{1 i} \\ 0 & c_{1 i}\end{array}\right)$ for $i=1,2,3$ belonging to $U_{2}(G)$ with entries being generators of $G$. It is easy to see that

$$
\left[X_{1}, X_{2}, X_{3}\right]=\left(\begin{array}{cc}
{\left[a_{11}, a_{12}, a_{13}\right]} & * \\
0 & {\left[c_{11}, c_{12}, c_{13}\right]}
\end{array}\right)=\left(\begin{array}{cc}
0 & * \\
0 & 0
\end{array}\right) .
$$

The same form has the matrix $\left[X_{4}, X_{5}, X_{6}\right]$. As the only possibly non-zero entry of the considered matrices is the $(1,2)$-entry, the multiplication gives 0 . 
Remark 3. Theorem 6 could be reformulated as follows. Let $f(x, y, z)=[x, y, z]$. The $2 \times 2$ upper triangular matrices over the Grassmann algebra satisfy the identity $f^{2}=0$ while their entries satisfy the identity $f=0$.

In [3, Theorem 3.1], Domokos gave a compact form of a theorem of Szigeti from [15], namely,

Proposition 3. For any $2 \times 2$ matrix $X$ over a $K$-algebra $S$ satisfying the identity $\left[x_{1}, x_{2}, x_{3}\right]=0$ we have that

$$
\begin{aligned}
X^{4}- & 2 X^{3}(\operatorname{tr} X)+X^{2}\left(2 \operatorname{tr}^{2} X-\operatorname{tr} X^{2}\right)+X\left(\frac{1}{2} \operatorname{tr} X \circ \operatorname{tr} X^{2}-\operatorname{tr}^{3} X\right) \\
+ & \frac{1}{4}\left(\operatorname{tr}^{4} X+\operatorname{tr}^{2} X^{2}+\frac{1}{2} \operatorname{tr}^{2} X \operatorname{tr} X^{2}-\frac{5}{2} \operatorname{tr} X^{2} \operatorname{tr}^{2} X+2\left[\operatorname{tr} X^{3}, \operatorname{tr} X\right]\right) E
\end{aligned}
$$

and

$$
\begin{aligned}
X^{4}- & 2(\operatorname{tr} X) X^{3}+\left(2 \operatorname{tr}^{2} X-\operatorname{tr} X^{2}\right) X^{2}+\left(\frac{1}{2} \operatorname{tr} X \circ \operatorname{tr} X^{2}-\operatorname{tr}^{3} X\right) X \\
& +\frac{1}{4}\left(\operatorname{tr}^{4} X+\operatorname{tr}^{2} X^{2}-\frac{5}{2} \operatorname{tr}^{2} X \operatorname{tr} X^{2}+\frac{1}{2} \operatorname{tr} X^{2} \operatorname{tr}^{2} X-2\left[\operatorname{tr} X^{3}, \operatorname{tr} X\right]\right) E
\end{aligned}
$$

are equal to zero in $S^{2 \times 2}$.

In [15], Szigeti developed a new theory of determinants of $n \times n$ matrices over rings satisfying the polynomial identity of $m$-Lie nilpotency

$$
\left.\left[\left[\left[\cdots\left[x_{1}, x_{2}\right], x_{3}\right], \cdots\right], x_{m}\right], x_{m+1}\right]=0 .
$$

As the Grassmann algebra is 2-Lie nilpotent the defined in [15] right $m$-adjoint of a matrix, the right $m$-determinant of a matrix $r d_{m}$ and the right $m$-characteristic polynomial $p(x)$ of a matrix and their properties could be interpreted for the matrix algebra $U_{2}(G)$.

Proposition 4 ([15, Theorem 4.2]). If $p(x)=\lambda_{0}+\lambda_{1} x+\cdots+\lambda_{d} x^{d}$ is the right $m$-characteristic polynomial of a $n \times n$ matrix $A \in M_{n}(R)$ over a $m$-Lie nilpotent ring $R$ then the left substitution of $A$ into $p(x)$ is zero: $(A) p=E \lambda_{0}+A \lambda_{1}+\cdots+$ $A^{d} \lambda_{d}=0$.

Again in [15], Szigeti pointed out the identity of "algebraicity" for matrices over the Grassmann algebra.

Proposition 5 ([15, Theorem 5.1]). The polynomial identity

$$
S_{2 n^{2}}\left(\left[Y^{2 n^{2}}, Z\right],\left[Y^{2 n^{2}-1}, Z\right], \ldots,\left[Y^{2}, Z\right],[Y, Z]\right)=0
$$

holds on $M_{n}(G)$ for any two matrices $Y$ and $Z$.

Now we give some laws and identities for the upper triangular matrices over the Grassmann algebra $G$. 
Theorem 7. Let the matrix $X=\left(\begin{array}{cc}a & b \\ 0 & c\end{array}\right)$ belong to $U_{2}(J(G))$ for $a, b, c$ being basic monomials of $G^{\prime}$.

(I) The following laws are valid for $X$ :

$$
\begin{aligned}
(X-a)(X-c) & =0, \\
X^{3}(\operatorname{tr} X) & =0, \\
(\operatorname{tr} X) X^{3} & =0 .
\end{aligned}
$$

(II) Two identities hold for any matrices $X$ and $Y$ of the considered type, namely $X^{2} Y^{2}=0$ and $\left(X^{2} Y\right)^{2}=0$.

Thus any matrix $X$ is nilpotent of index 4 . A matrix $X$ with $\operatorname{tr} X=0$ is nilpotent of index 3. $X$.

Proof. (I) Direct calculations give the validity of the three stated laws for a matrix

(II) Again applying direct calculations we get that the only non-zero entries in $X^{2}, Y^{2}$ and $X^{2} Y$ are the $(1,2)$ entries and the corresponding multiplication gives zero.

We see that both Proposition 3 and Proposition 4 for $n=2$ are compatible with Theorem 7 as for such a matrix $A \in U_{2}(G)$ we have $\operatorname{rdet}_{2} A=0$ and $p(x)=\operatorname{rdet}_{2}(A-$ $E x)=x^{4}-2 \operatorname{rdet} A x^{3}$. Thus $A^{4}-2(\operatorname{tr} A) A^{3}=0$.

Corollary 1. An identity of degree 9 holds for any two matrices $Y$ and $Z$ from $U_{2}(G)$ with entries being basic monomials, namely, $S_{3}\left(\left[Y^{3}, Z\right],\left[Y^{2}, Z\right],[Y, Z]\right)=0$.

Proof. Applying Proposition 5 and the index of nilpotency of the matrix $Y$.

Remark 4. If we consider the Grassmann algebra over a finite dimensional space, the corresponding identities have much smaller degrees.

Theorem 8. The following two assertions hold:

(I) On the algebra $U_{2}\left(J\left(G_{2}\right)\right)$ we get the identity $X Y Z=0$ (respectively, $X^{3}=$ $0)$.

(II) Any matrix $X=\left(\begin{array}{cc}a & b \\ 0 & c\end{array}\right)$ from $U_{2}\left(J\left(G_{2}\right)\right)$ satisfies the law $(\operatorname{tr} X) X^{2}=0$, respectively, $X^{2}(\operatorname{tr} X)=0$.

Proof. In the considered algebra the square of every element is zero. The identity and the law are proved directly.

Multiplying two linear combinations of the basic elements $e_{1}, e_{2}$ and $e_{1} e_{2}$ we get only $\alpha e_{1} e_{2}$. Its product with any other linear combination of $e_{1}, e_{2}$ and $e_{1} e_{2}$ gives zero.

Analogous considerations are valid for the linear combinations of the basic elements of any finite dimensional Grassmann algebra (over a $n$-dimensional vector 
space). The multiplication of $n$ linear combinations will result in $\alpha e_{1} e_{2} \ldots e_{n}$ and the result of the next multiplication will be zero. Thus, we come to the following

Corollary 2. The matrix algebra $U_{k}\left(J\left(G_{n}\right)\right)$ (respectively, $M_{k}\left(J\left(G_{n}\right)\right)$ ) is nilpotent of class $\leq n+1$.

Corollary 3. The polynomial identity $S_{n-1}\left(\left[Y^{n-1}, Z\right],\left[Y^{n-2}, Z\right], \ldots,[Y, Z]\right)=0$ holds on $M_{k}\left(J\left(G_{n}\right)\right)$ for $n \geq 3$ and $k \geq 2$.

As the algebra $U_{2}(G)$ is a subalgebra of $M_{2}(G)$ we turn to the Hall identity, the four degree standard identity for $M_{2}(K)$ and the product commutator identity for $U_{2}(K)$ considering the Grassmann algebra $G$ instead of the field $K$. We get

Theorem 9. The polynomials $\left[\left[x_{1}, x_{2}\right]^{2}, x_{1}\right], S_{4}\left(x_{1}, x_{2}, x_{3}, x_{4}\right)$, and $\left[x_{1}, x_{2}\right]\left[x_{3}, x_{4}\right]$ are not identities for the algebra $U_{2}(G)$.

Proof. A counter example for the validity of the first and the third identity gives the matrices

$$
X_{1}=\left(\begin{array}{cc}
e_{1} & e_{2} \\
0 & e_{3}
\end{array}\right)
$$

and

$$
X_{2}=\left(\begin{array}{cc}
e_{4} e_{5}+e_{6} & 1 \\
0 & e_{1}+e_{3}
\end{array}\right)
$$

We get that the $(1,2)$-entry of $\left[x_{1}, x_{2}\right]^{2}$ is nonzero, namely,

$$
2 e_{1} e_{3} e_{6}+2 e_{1} e_{2} e_{4} e_{5} e_{6}-2 e_{1} e_{2} e_{3} e_{4} e_{5}+4 e_{1} e_{2} e_{3} e_{6} .
$$

The $(1,2)$-entry of $\left[\left[x_{1}, x_{2}\right]^{2}, x_{1}\right]$ is $-2 e_{1} e_{2} e_{3} e_{4} e_{5} e_{6}$. For the second statement, we rely on the general case considered later.

In [1,2], a connection is given between the identities on $M_{n}(K)$ and those on $M_{n}(G)$.

Proposition 6 ([2, Proposition 2.1]). Let $f_{1}, \ldots, f_{d} \in K\left\langle x_{1}, \ldots, x_{m}\right\rangle$ be elements of the T-ideal of identities of $M_{n}(K)$. If $d>\frac{1}{2} n^{2} m$, then $f_{1} f_{2} \cdots f_{d}=0$ is an identity on $M_{n}(G)$.

Remark 5. Applying the result to $M_{2}(G)$ and the standard polynomial $S_{4}$ we get that $S_{4}^{9}=0$ is an identity on $M_{2}(G)$. However, this is not the best possible result. Really on $M_{2}(G)$ we get the identities $S_{4}^{5}=0$ and $\left[[x, y]^{2}, x\right]^{5}=0$. Thus we get two identities of degree 20 and 25, respectively, for $U_{2}(G)$.

The above Proposition 6 has an analogue for the upper triangular matrices $U_{n}$.

Theorem 10. Let $f_{1}, \ldots, f_{d} \in K\left\langle x_{1}, \ldots, x_{m}\right\rangle$ be elements of the $T$-ideal of identities of $U_{n}(K)$. If $d>\frac{1}{4} n(n+1) m$, then $f_{1} f_{2} \cdots f_{d}=0$ is an identity on $U_{n}(G)$. 
Proof. It follows the proof of Proposition 6 taking into account the dimension of $U_{n}(K)$ and the fact that the relatively free algebra $F\left(U_{n}(K)\right)$ has as a basis the monomials

$$
x_{1}^{a_{1}} \cdots x_{m}^{a_{m}}\left[x_{i_{11}}, x_{i_{21}}, \ldots, x_{i_{p_{1}}}\right] \cdots\left[x_{i_{1 r}}, x_{i_{2 r}}, \ldots, x_{i_{p r}}\right],
$$

where the number $r$ of participating commutators is $\leq n-1$ and the indices in each commutator $\left[x_{i_{1 s}}, x_{i_{2 s}}, \ldots, x_{i_{p s} s}\right]$ satisfy the relations $i_{1 s}>i_{2 s} \leq \cdots<i_{p_{s} s}$.

Proposition 7 ([1, Lemma, p. 1509]). The algebra $M_{n}(G)$ satisfies the identity $S_{2 n}^{k}$ for some $k>1$ but satisfies neither $S_{2 n}$ nor identities of the form $S_{m}^{k}$ for any $k$ when $m<2 n$.

Theorem 11. The algebra $U_{n}(G)$ does not satisfy the identity $S_{2 n}=0$.

Proof. We have

$$
S_{2 n}\left(e_{11}, e_{12}, e_{22}, e_{23}, \ldots, e_{n-1, n-1}, e_{n-1, n}, e e_{n n}, f e_{n n}\right)=2 e f e_{1 n} \neq 0
$$

for $e, f \in G$ such that $e f=-f e \neq 0$.

In [16, Proposition 4.1 and Corollary 6.1], Vishne described an efficient way to use the $S_{n}$-module structure in the computation of the multilinear identities of degree $n$ of a given algebra. He used the method to show that the minimal degree of an identity for $M_{2}(G)$ is 8 and gave explicit identities of degree 8 . He described a class of identities for $M_{2}(G)$, namely,

Proposition 8 ([16, Corollary 4.3]). Let $f$ be a multilinear polynomial of degree 8. If $\operatorname{tr} f\left(x_{\sigma(1)}, \ldots, x_{\sigma(8)}\right)=0$ for every $x_{1}, \ldots, x_{8} \in M_{2}(G)$, then $f$ is an identity of $M_{2}(G)$.

We use the notation $A_{n}=\sum_{\sigma \in \operatorname{Sym}(n)} x_{\sigma(1)} x_{\sigma(2)} \cdots x_{\sigma(n)}$. By $G_{0}^{\prime}$ we denote the even part of $G^{\prime}$ and by $G_{1}^{\prime}$ its odd part.

Proposition 9. The following identities hold:

(1) On $U_{2}\left(G_{1}^{\prime}\right)$ we have $A_{k}^{2}=0$ for every integer $k$.

(2) On $U_{2}\left(G_{0}^{\prime}\right)$ we have $S_{k}^{2}=0$ for every integer $k$.

Proof. For $x_{1}, x_{2} \in U_{2}\left(G_{1}^{\prime}\right)$ and $A_{2}=\left(a_{i j}\right)$ we get $a_{11}=a_{21}=a_{22}=0$. Thus $A_{2}^{2}=0$. Then we use induction. Let $A_{k-1}\left(x_{1}, \ldots, x_{k-1}\right)$ have only one nonzero entry, namely the $(1,2)$-entry. We have

$$
\begin{aligned}
A_{k}\left(x_{1}, \ldots, x_{k}\right)= & A_{k-1}\left(x_{1}, \ldots, x_{k-1}\right) x_{k}+A_{k-1}\left(x_{1}, \ldots, x_{k-2}, x_{k}\right) x_{k-1} \\
& +A_{k-1}\left(x_{1}, \ldots, x_{k-3}, x_{k-1}, x_{k}\right) x_{k-2}+\cdots \\
& +A_{k-1}\left(x_{1}, x_{3}, \ldots, x_{k}\right) x_{2}+A_{k-1}\left(x_{2}, \ldots, x_{k}\right) x_{1} .
\end{aligned}
$$

The multiplication by $x_{i}$ keeps the three zero entries in every summand. So for $A_{k}=\left(b_{i j}\right)$ we have $b_{11}=b_{21}=b_{22}=0$ and thus $A_{k}^{2}=0$. 
The arguments for $S_{2}^{2}=0$ are similar as for $x_{1}, x_{2} \in U_{2}\left(G_{0}^{\prime}\right)$ and for $S_{2}=\left(c_{i j}\right)$ we have $c_{11}=c_{21}=c_{22}=0$. The recursive formulas

$$
S_{k}\left(x_{1}, \ldots, x_{k}\right)=\sum_{i=1}^{k} x_{i}(-1)^{k-1} S_{k-1}\left(x_{i+1}, \ldots, x_{k}, x_{1}, \ldots, x_{i-1}\right)
$$

for $k$ even and

$$
S_{k}\left(x_{1}, \ldots, x_{k}\right)=\sum_{i=1}^{k} x_{i} S_{k-1}\left(x_{i+1}, \ldots, x_{k}, x_{1}, \ldots, x_{i-1}\right)
$$

for $k$ odd show that for $S_{k}=\left(d_{i j}\right)$ we have $d_{11}=d_{21}=d_{22}=0$ and, therefore, $S_{k}^{2}=0$.

\section{ACKNOWLEDGEMENT}

The authors give their thanks to Professor Jenô Szigeti for his hospitality and useful discussions made concerning the text during the authors' stay at the University of Miskolc in the summer of 2008.

\section{REFERENCES}

[1] A. Berele, "Classification theorems for verbally semiprime algebras," Comm. Algebra, vol. 21, no. 5, pp. 1505-1512, 1993.

[2] M. Domokos and A. Popov, "On the degree of nilpotency of the radical of relatively free algebras," Math. Pannon., vol. 8, no. 1, pp. 11-16, 1997.

[3] M. Domokos, "Cayley-Hamilton theorem for $2 \times 2$ matrices over the Grassmann algebra," J. Pure Appl. Algebra, vol. 133, no. 1-2, pp. 69-81, 1998, ring theory (Miskolc, 1996).

[4] V. Drensky, Free algebras and PI-algebras. Springer-Verlag Singapore, Singapore, 2000, graduate course in algebra.

[5] A. R. Kemer, Ideals of identities of associative algebras, ser. Translations of Mathematical Monographs. Providence, RI: American Mathematical Society, 1991, vol. 87, translated from the Russian by C. W. Kohls.

[6] D. Krakowski and A. Regev, "The polynomial identities of the Grassmann algebra," Trans. Amer. Math. Soc., vol. 181, pp. 429-438, 1973.

[7] D. La Mattina and P. Misso, "Algebras with involution with linear codimension growth," J. Algebra, vol. 305, no. 1, pp. 270-291, 2006.

[8] K. R. Pearson, "A lower bound for the degree of polynomials satisfied by matrices," J. Austral. Math. Soc. Ser. A, vol. 27, no. 4, pp. 430-436, 1979.

[9] K. R. Pearson, "Degree 7 monic polynomials satisfied by a $3 \times 3$ matrix over a noncommutative ring," Comm. Algebra, vol. 10, no. 19, pp. 2043-2073, 1982.

[10] K. R. Pearson, "The Robson cubics generate all polynomials satisfied by the general $2 \times 2$ matrix," Comm. Algebra, vol. 10, no. 19, pp. 2075-2084, 1982.

[11] T. Rashkova, "The Robson cubics for matrix algebras with involution," Acta Univ. Apulensis Math. Inform., no. 15, pp. 341-351, 2008.

[12] J. C. Robson, "Polynomials satisfied by matrices," J. Algebra, vol. 55, no. 2, pp. 509-520, 1978.

[13] J. C. Robson, "Well quasi-ordered sets and ideals in free semigroups and algebras," J. Algebra, vol. 55, no. 2, pp. 521-535, 1978. 
[14] J. C. Robson, "Generators of the polynomials satisfied by matrices," in Ring theory (Proc. Antwerp Conf. (NATO Adv. Study Inst.), Univ. Antwerp, Antwerp, 1978), ser. Lecture Notes in Pure and Appl. Math. New York: Dekker, 1979, vol. 51, pp. 243-255.

[15] J. Szigeti, "New determinants and the Cayley-Hamilton theorem for matrices over Lie nilpotent rings," Proc. Amer. Math. Soc., vol. 125, no. 8, pp. 2245-2254, 1997.

[16] U. Vishne, "Polynomial identities of $M_{2}(G)$," Comm. Algebra, vol. 30, no. 1, pp. 443-454, 2002.

Authors' addresses

T. Rashkova

University of Russe “A. Kanchev”, Department of Algebra and Geometry, 8 Studentska St., 7017 Russe, Bulgaria

E-mail address: tsrashkova@ru.acad.bg

A. Mihova

University of Russe "A. Kanchev", Department of Mathematical Analysis, 8 Studentska St., 7017 Russe, Bulgaria

E-mail address: amihova@ru.acad.bg 